\title{
Association between sensitization and allergic diseases in 7-years-old Korean children
}

Eun Lee, ${ }^{1}$ Dong In Suh, ${ }^{2}$ So-Yeon Lee, ${ }^{3}$ Sungsu Jung, ${ }^{4}$ Jisun Yoon, ${ }^{5}$ Hyun-Ju Cho, ${ }^{6}$ Youngho Kim, ${ }^{3}$ Song-I Yang, ${ }^{7}$ Ji-won Kwon, ${ }^{8}$ Gwang Cheon Jang, ${ }^{9}$ Yong Han Sun,${ }^{10}$ Sung-Il Woo, ${ }^{11}$ You-Sook Youn, ${ }^{12}$ Kang Seo Park, ${ }^{13}$ Hwa Jin Cho, ${ }^{1}$ Myung-Hee Kook, ${ }^{14}$ Hye Ryoung Yi, ${ }^{14}$ Hai Lee Chung, ${ }^{15}$ Ja-Hyeong Kim, ${ }^{16}$ Hyung Young Kim, ${ }^{4}$ Jin A Jung, ${ }^{17}$ Hyang-Ok Woo, ${ }^{18}$ Jeom-Kyu Lee, ${ }^{19}$ Woo-Sung Chang, ${ }^{19}$ Soo-Jong Hong ${ }^{3}$

\section{Abstract}

Background: Sensitization is associated with the exacerbation, severity, and prognosis of allergic diseases in children

Objective: We characterized the association between sensitization patterns and allergic diseases.

Methods: A cohort of 548 children was enrolled from Panel Study of Korean Children (PSKC) study. Skin prick tests (SPTs) for 18 common allergens, blood tests, and methacholine bronchial challenge tests were performed at age 7 . The Korean version of International Study of Asthma and Allergies in Childhood (ISAAC) questionnaire was used.

Results: The sensitization rate on SPTs was $46.4 \%$. Sensitization to indoor allergens showed an association with symptoms of asthma (adjusted odds ratio [aOR], 2.39; 95\% confidence intervals [95\% CIs], 1.10-5.23), allergic rhinitis (AR, aOR 2.08, 95\% CIs 1.42-3.06), and atopic dermatitis (AD, aOR 2.36, 95\% CIs 1.24-4.50) in the preceding 12 months. In contrast, sensitization to outdoor allergens was associated with AR diagnosis only (aOR 2.40, 95\% CIs 1.30-4.41). The number of sensitized allergens was associated with a lifetime diagnosis and symptoms in the preceding 12 months of $\mathrm{AR}$ and asthma, but not with $\mathrm{AD}$ or BHR. A higher degree of sensitization to indoor allergens was associated with symptoms in the preceding 12 months of asthma, AR, AD, and that for outdoor allergens was associated with symptoms in the prior 12 months of asthma and AR.

Conclusion: The sensitization patterns including allergen type, number, and degree of sensitization are helpful for interpreting the association between sensitization and allergic diseases and identifying the pathophysiologies and diverse phenotypes of allergic diseases.

Key words: sensitization; pattern; children; allergic diseases; bronchial hyperresponsiveness.

\section{Affiliations:}

${ }^{1}$ Department of Pediatrics, Chonnam National University Hospital, Chonnam National University Medical School, Gwangju, Korea

${ }^{2}$ Department of Pediatrics, Seoul National University College of Medicine, Seoul, Korea

Department of Pediatrics, Childhood Asthma Atopy Center, Environmental Health Center, Asan Medical Center, University of Ulsan College of Medicine, Seoul, Korea

${ }^{4}$ Department of Pediatrics, Pusan National University Yangsan Hospital, Yangsan, Republic of Korea

Department of Pediatrics, Mediplex Hospital, Incheon, Republic of Korea

${ }^{6}$ Department of Pediatrics, International St. Mary's hospital, Catholic Kwandong University, Incheon, Korea

Department of Pediatrics, Hallym University Sacred Heart Hospital, Hallym University College of Medicine, Anyang, Korea

${ }^{8}$ Department of Pediatrics, Seoul National University Bundang Hospital, Seongnam, Korea

${ }^{9}$ Department of Pediatrics, National Health Insurance Service Ilsan Hospital, Ilsan, Korea
${ }^{10}$ Department of Pediatrics, Gachon University Gil Medical Center, Incheon, Korea

${ }^{11}$ Department of Pediatrics, College of Medicine, Chungbuk National University, Cheongju, Korea

${ }^{12}$ Departments of Pediatrics, College of Medicine,

The Catholic University of Korea, Daejeon, Korea

${ }^{13}$ Department of Pediatrics, Presbyterian Medical Center, Jeonju, Korea

${ }^{14}$ Department of Pediatrics, Gwangju Veterans Hospital, Gwangju, Korea

${ }^{15}$ Department of Pediatrics, Catholic University of Daegu School of Medicine, Daegu, Korea

${ }^{16}$ Department of Pediatrics, Ulsan University Hospital, Ulsan, Korea

${ }^{17}$ Department of Pediatrics, Anatomy, College of Medicine, Dong-A University, Busan, Korea

${ }^{18}$ Department of Pediatrics, Gyeongsang National University College of Medicine, Jinju, Korea

${ }^{19}$ Division of Allergy and Chronic Respiratory Diseases, Center for Biomedical Sciences Korea National Institute of Health, Korea Centers for Disease Control and Prevention, Osong, Korea 
Corresponding author:

Soo-Jong Hong

Department of Pediatrics, Childhood Asthma Atopy Center,

Asan Medical Center College of Medicine, Ulsan University

88 Olympic-ro 43 gil, Songpa-gu, Seoul 05505, Korea

E-mail: sjhong@amc.seoul.kr

\section{Introduction}

Sensitization is associated with development, severity, and prognosis of allergic diseases in children. ${ }^{1}$ Diverse factors including age, ethnicity, time, region, and combined allergic diseases affect allergic sensitization..$^{2-4}$ Among these variables, age and combined allergic diseases have shown the strongest association with allergic sensitization. ${ }^{3,5-11}$

The sensitization rates change over times., ${ }^{9,12}$ In a previous nationwide cross-sectional study from Korea in 2010, the sensitization rate was $47.9 \%$ in 6-7 year-old children. ${ }^{12}$ Recent studies on changes in the prevalence of sensitization are relatively lacking, compared to the numerous previous studies; ${ }^{13,14}$ which would be helpful in identifying the role of sensitization in allergic diseases and establishing new preventive strategies for these conditions in children.

Sensitization patterns affect the prognosis of allergic diseases, such as the new development of asthma and bronchial hyperresponsiveness (BHR). ${ }^{11}$ The type of sensitization may also play a different role according to allergic diseases. ${ }^{6,15}$ Notably, however previous studies have been limited in identifying the association between allergic diseases and specific sensitization patterns due to the confounding nature of multi-sensitization.

In our present study, we investigated the prevalence of sensitization to common allergens in a cohort of children who were aged 7 years in 2015 and evaluated how the sensitization pattern is associated with allergic diseases in these subjects. We elucidated this association in terms of the allergen types, number of sensitized allergens, and degree of sensitization.

\section{Materials and Methods Study population}

The Children Panel Study is a nationwide hospital-based birth cohort study that began in 2008. A total of 2,150 mothers were recruited at 30 gynecology clinics selected by random sampling. This study was based on responses to questionnaires administered by individual interviewers in each year. Among the enrolled children, 1,577 children were followed up at 7 years of age. Parents and their children, who agreed to undergo allergy tests, visited hospitals. The methacholine challenge test (MCT) was performed in 565 children, and skin prick tests (SPTs) were performed in 548 children at the age of 7 years. These tests were performed in a day at hospitals with an additional questionnaire on the presence of allergic diseases. From the total panel population, we enrolled 548 children in this current study for whom the results of SPTs were available. There were no significant differences in the baseline characteristics between the included and excluded subjects. The Institutional Review Board of Asan Medical Center reviewed and approved the current study protocol (IRB no. 2015-0907). Written consent was obtained from the parents or guardians of each subject following a detailed explanation of the study.

\section{Skin prick tests (SPTs)}

SPTs were performed for 18 common allergens. Histamine $(10 \mathrm{mg} / \mathrm{ml})$ was used as the positive control, and normal saline was used as the negative control. Positive sensitization was defined as a mean wheal diameter of $\geq 3 \mathrm{~mm}$ and a greater size than that elicited by the positive control when measured after $15 \mathrm{~min}$. Atopy was defined as at least one positive SPT response. The prevalence of sensitization to food, including peanut, milk, and egg white, was very low and was thus not considered in our current analyses. The 15 allergens evaluated in the test were classified into 3 groups to identify the association between specific sensitization and development of allergic diseases. These included indoor allergens (Dermatophagoides farinae [Der f], Dermatophagoides pteronyssinus [Der p], cat epithelium, dog dander, cockroach), outdoor allergens (grass mixture, alder, birch, oak, Japanese hop, mugwort, ragweed, hazel) and mold (Alternaria, Aspergillus). In the binary classification into indoor and outdoor allergens, Alternaria was included in the outdoor allergen group and Aspergillus was classified as an indoor allergen. ${ }^{16}$ Mono-sensitization was defined as sensitization to only one inhalant allergen and poly-sensitization to more than two inhalant allergens. The sensitization grading we used with the SPTs was as described previously: grade 0 , ratio of allergen wheal to histamine $(\mathrm{R})=0$; grade $1,<1 / 2$; grade $2, \geq 1 / 2$ to $<1$; grade 3,1 $\geq$ to $<2$; grade $4,2 \geq$ to $<3$; grade $5,3 \geq$ to $<4$; and grade 6 , $\geq 4 .^{17}$

\section{Methacholine challenge test (MCT)}

For MCT, we used a modified five-breath dosimeter method in accordance with the American Thoracic Society (ATS) guidelines. ${ }^{18}$ Spirometry (Jaeger APS; CareFusion Respiratory Care, San Diego, CA) was conducted using a Hans-Rudolph non-rebreathing valve (Hand Rudolph Inc., Kansas City, MO) and a Misty-Neb Medication nebulizer set (CareFusion Respiratory Care) with the flow meter set at $0.009 \mathrm{~mL} \pm 10 \%$ of the solution per $0.6 \mathrm{~s}$ of actuation during inhalation. Subjects were excluded from the analysis if they had a history of upper or lower respiratory tract infection during the three weeks prior to MCT or had received inhaled corticosteroid therapy at least four weeks before MCT. Normal saline was used as a baseline and was followed by stepwise concentrations of methacholine concentrations $(0.0625,0.25,1,4$, and $16 \mathrm{mg} / \mathrm{mL})$. The forced expiratory volume in 1 second $\left(\mathrm{FEV}_{1}\right)$ was measured at $30 \mathrm{~s}$ and $90 \mathrm{~s}$ after the nebulization was completed and the next dosing schedule then proceeded within 5 minutes. We defined BHR to methacholine in our current series in a dual way i.e. we adopted the PC20 cutoff $<8 \mathrm{mg} / \mathrm{mL}^{18}$

\section{ISAAC Questionnaire}

The Korean version of the International Study of Asthma and Allergies in Childhood (ISAAC) questionnaire was used to investigate asthma, allergic rhinitis (AR), and atopic dermatitis (AD). ${ }^{19}$ The parents or guardians of the subjects completed this questionnaire, which includes the following items: (i) general characteristics, such as sex, age, height, and weight; (ii) history of $\mathrm{AD}$ diagnosis by physicians or $\mathrm{AD}$ symptoms in the last 12 months, history of AR diagnosis by physicians 
or AR symptoms in the last 12 months, history of doctor-diagnosed asthma, and the presence of wheezing in the last 12 months; and (iii) household income and environmental factors, such as exposure to environmental tobacco smoke (ETS).

\section{Statistical analysis}

The associations between sensitization, diverse environmental factors, and allergic diseases were analyzed using multivariate logistic regression analysis and expressed as an odds ratio (OR) with a 95\% confidence interval (CI). Adjustments were made for potential confounding factors, such as sex, living areas, parental history of allergic diseases, exposure to ETS, and monthly household income. All statistical analyses were performed using SPSS version 24.0 (IBM Co., Armonk, NY). A $P$ value of less than 0.05 was set as the level of statistical significance.

\section{Results}

\section{Characteristics of the study population}

The characteristics of the study population are listed in Table 1 . The mean age of the subjects was $7.0 \pm 0.1$ years old and $53.8 \%$ of the total study population was male. In addition, $67.9 \%$ of the study population had a family history of allergic diseases.

Table 1. Characteristics of the enrolled population in the present study

\begin{tabular}{|lc|}
\hline \multicolumn{1}{c}{ Variables } & N (\%) or mean \pm SD \\
\hline Age (years) & $7.0 \pm 0.1$ \\
\hline Sex, male & $296 / 548(53.8)$ \\
\hline Gestational age (days) & $274.0 \pm 9.0$ \\
\hline Breast feeding & $251 / 514(48.8)$ \\
\hline Delivery mode, vaginal delivery & $313 / 536(58.4)$ \\
\hline Maternal age at delivery (years) & $31.4 \pm 3.6$ \\
\hline Parental history of allergic diseases & $353 / 520(67.9)$ \\
\hline Exposure to environmental tobacco smoke & $348 / 546(63.7)$ \\
\hline Monthly household income & \\
\hline$<300 \$$ & $221(40.5)$ \\
\hline $300 \$ \leq$ income $<500 \$$ & $222(40.5)$ \\
\hline
\end{tabular}

$\mathrm{N}$, number; SD, standard deviation.

\section{Association between sensitization rate and allergic diseases}

Children with symptoms of $\mathrm{AR}$, asthma, and $\mathrm{AD}$ in the preceding 12 months as well as BHR showed a significantly higher sensitization rate (Table 2). Children diagnosed with $\mathrm{AR}$, asthma, or $\mathrm{AD}$ in their lifetime also showed significantly higher sensitization rates compared to those without a diagnosis of any allergic diseases by 7 years of age.
Table 2. Sensitization rates according to different allergic diseases

\begin{tabular}{|c|c|c|c|}
\hline Variables & $\begin{array}{c}\text { Non-atopy, } \\
\mathbf{N}(\%) \\
(\mathbf{n}=294)\end{array}$ & $\begin{array}{c}\text { Atopy, } \\
\mathbf{N}(\%) \\
(\mathbf{n}=254)\end{array}$ & $P$ value \\
\hline AR diagnosis ever & $\begin{array}{c}136 / 289 \\
(47.1)\end{array}$ & $\begin{array}{c}166 / 250 \\
(66.4)\end{array}$ & $<0.001$ \\
\hline $\begin{array}{l}\text { AR symptom in the pre- } \\
\text { ceding } 12 \text { months }\end{array}$ & $\begin{array}{c}127 / 290 \\
(43.8)\end{array}$ & $\begin{array}{c}161 / 250 \\
(64.4)\end{array}$ & $<0.001$ \\
\hline $\begin{array}{l}\text { Asthma diagnosis in } \\
\text { lifetime }\end{array}$ & $\begin{array}{c}15 / 289 \\
(5.2)\end{array}$ & $\begin{array}{c}28 / 250 \\
(11.2)\end{array}$ & 0.011 \\
\hline $\begin{array}{l}\text { Asthma symptoms in the } \\
\text { preceding } 12 \text { months }\end{array}$ & $\begin{array}{c}9 / 295 \\
(3.1)\end{array}$ & $\begin{array}{c}18 / 253 \\
(7.1)\end{array}$ & 0.018 \\
\hline $\mathrm{AD}$ diagnosis in lifetime & $\begin{array}{l}61 / 291 \\
(21.0)\end{array}$ & $\begin{array}{c}79 / 251 \\
(31.5)\end{array}$ & 0.006 \\
\hline $\begin{array}{l}\text { AD symptoms in the } \\
\text { preceding } 12 \text { months }\end{array}$ & $\begin{array}{l}34 / 295 \\
(11.5)\end{array}$ & $\begin{array}{l}49 / 253 \\
(19.4)\end{array}$ & 0.033 \\
\hline $\mathrm{BHR}<8 \mathrm{mg} / \mathrm{mL}$ & $\begin{array}{l}28 / 263 \\
(10.6)\end{array}$ & $\begin{array}{l}46 / 220 \\
(20.9)\end{array}$ & 0.002 \\
\hline
\end{tabular}

Chi-square test

$\mathrm{AD}$, atopic dermatitis; $\mathrm{AR}$, allergic rhinitis; BHR, bronchial hyperresponsiveness.

\section{Sensitization rate of the study subjects to specific allergens}

We found that $46.4 \%$ of the children were sensitized to at least one of the common allergens at 7 years of age. When we stratified the sensitized allergens into indoor, outdoor and mold allergen groups, the study population was most commonly sensitized to the indoor allergens (31.8\%). Among the children sensitized to more than one allergen, $77.2 \%$ (196/254) were poly-sensitized and $22.8 \%$ (58/254) were mono-sensitized.

The most common sensitizations in the total population were to $\operatorname{Der} p(38.0 \%)$ and $\operatorname{Der} f(34.9 \%)$, followed by Japanese hop $(6.9 \%)$ and dog dander (4.4\%). The prevalence of sensitization was highest in the children with an asthma diagnosis in lifetime (66.7\%), followed by those with BHR (62.2\%). Children who had been diagnosed with $\mathrm{AD}$ and $\mathrm{AR}$ in their lifetime were most commonly sensitized to $\operatorname{Der} p$ and $\operatorname{Der} f$, followed by pollens, whereas those diagnosed with asthma showed the highest sensitization to $\operatorname{Der} p$ and $\operatorname{Der} f$, followed by dog dander and cat epithelium.

\section{Factors associated with sensitization at 7 years of age}

A parental history of any allergic disease showed an association with sensitization with an aOR of 1.84 (95\% CI, 1.25-2.71, $P=0.002$ ). Higher levels of maternal education were also associated with sensitization (aOR, 1.82; 95\% CI, $1.16-2.88, P=0.010$ ). Blood eosinophils of more than $4 \%$ and total serum IgE levels above $100 \mathrm{kU} / \mathrm{L}$ were also associated with allergen sensitization with an aOR of 4.35 (95\% CI, 2.946.46, $P<0.001)$ and 11.16 (95\% CI, 7.04-17.70, $P<0.001)$, respectively. 
Sensitization patterns in the study subjects stratified by allergic disease

Sensitization to indoor allergens showed an association with a prior diagnosis of $\mathrm{AD}, \mathrm{AR}$, and asthma as well as symptoms of these disorders in the preceding 12 months. On the other hand, sensitization to outdoor allergens was significantly associated with AR diagnosis in lifetime and symptoms in the preceding 12 months (Figure 1). Sensitization to mold was associated with wheezing and AR symptoms in the preceding 12 months. Sensitization to only indoor allergens was associated with BHR at 7 years of age.

To elucidate the independent effects of each sensitization groups on diverse allergic diseases, we stratified the sensitized allergens into house dust mite (HDM), pollen, mold and animal groups. We also evaluated the associations between sensitizations to diverse allergen groups and allergic diseases by classifying sensitizations to 'only other' group, defined as sensitizations to allergens other than the index allergen, and sensitizations to 'each + other' group, defined as sensitization to both index allergens and more than one of the other allergens in Figure 2. Sensitization to only HDM was significantly associated with recent symptoms of $\mathrm{AR}(\mathrm{aOR}, 2.10 ; 95 \%$ $\mathrm{CI}, 1.34-3.27), \mathrm{AD}(\mathrm{aOR}, 3.04$; 95\% CI, 1.40-6.59) and $\mathrm{BHR}$ (aOR, 2.74; 95\% CI, 1.48-5.05) (Figure 2). In terms of a lifetime diagnosis of any allergic disease, sensitization to only HDM was significantly associated with AR (aOR, 1.94; 95\% CI, 1.24-3.04). Simultaneous sensitizations to more than two allergen groups among the HDM, pollen, mold, and animal groups were significantly associated with asthma symptoms in the preceding 12 months (aOR, 3.69, 95\% CI, 1.46-9.23 for sensitization to HDM with other allergen groups; aOR, 3.35, 95\% CI, 1.21-9.33 for sensitization to pollen with other allergen groups; aOR, 10.74, 95\% CI, 1.95-59.30 for sensitization to mold with other allergen groups; aOR, 3.64, 95\% CI, 1.03 12.88 for sensitization to animal with other allergen groups;). Sensitization to mold in combination with other allergen groups showed a greater association with $\mathrm{BHR}$ at 7 years (aOR, 10.59; 95\% CI, 1.83-61.33), compared to sensitizations to only mold or sensitizations to combinations of allergens other than mold.

(A) Sensitization against indoor allergens

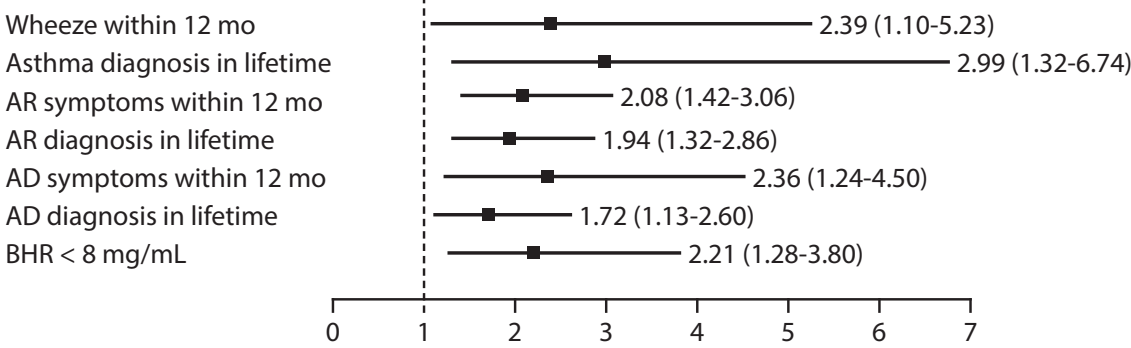

(B) Sensitization against outdoor allergens

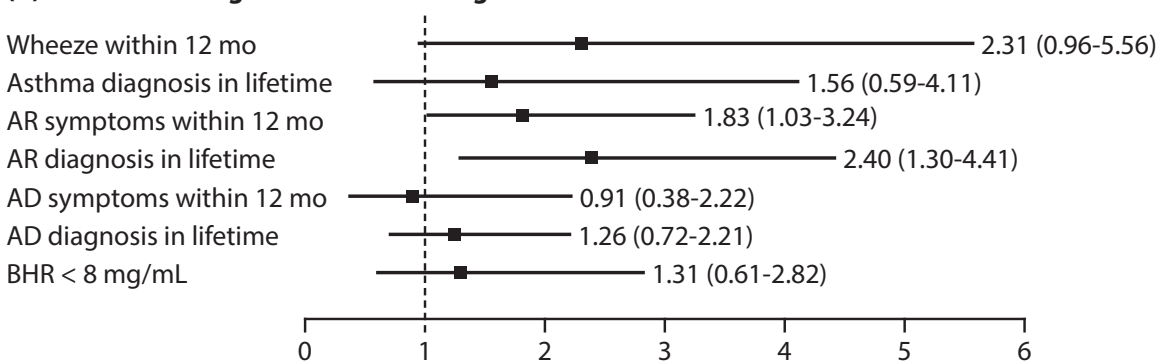

(C) Sensitization against mold

Wheeze within $12 \mathrm{mo}$ Asthma diagnosis in lifetime AR symptoms within 12 mo AR diagnosis in lifetime AD symptoms within $12 \mathrm{mo}$ $A D$ diagnosis in lifetime $\mathrm{BHR}<8 \mathrm{mg} / \mathrm{mL}$

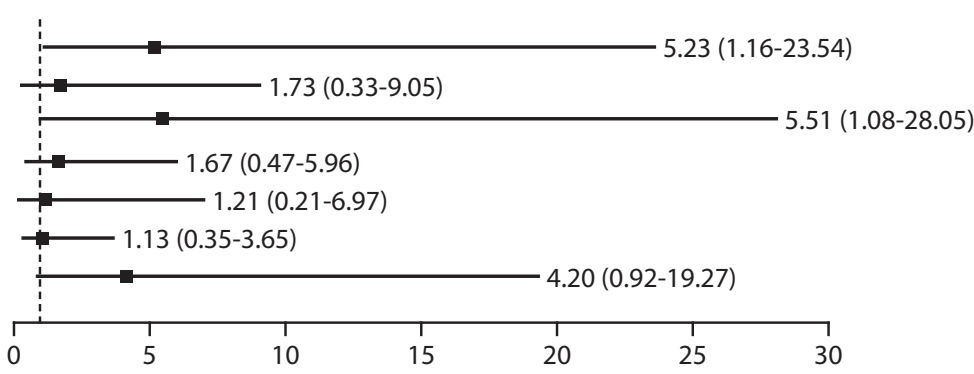

Figure 1. Adjusted odds ratio for allergic diseases in accordance with sensitizations against diverse allergen groups. (A) Association between the sensitization to indoor allergens and symptoms in the preceding 12 months and lifetime diagnosis of allergic diseases. (B) Association between the sensitization to outdoor allergens and symptoms in the preceding 12 months and lifetime diagnosis of allergic diseases. (C) Association between the sensitization to molds and symptoms in the preceding 12 months and lifetime diagnosis of allergic diseases.

Adjusted for sex, living areas, parental history of allergic diseases, and monthly household income. 
(A) Symptoms in the preceding 12 months

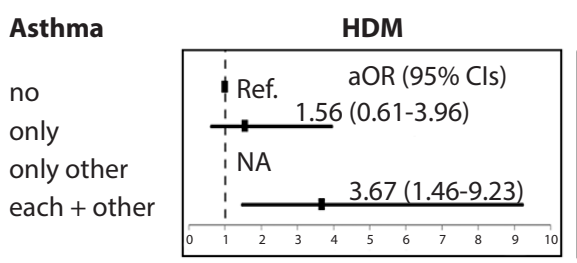
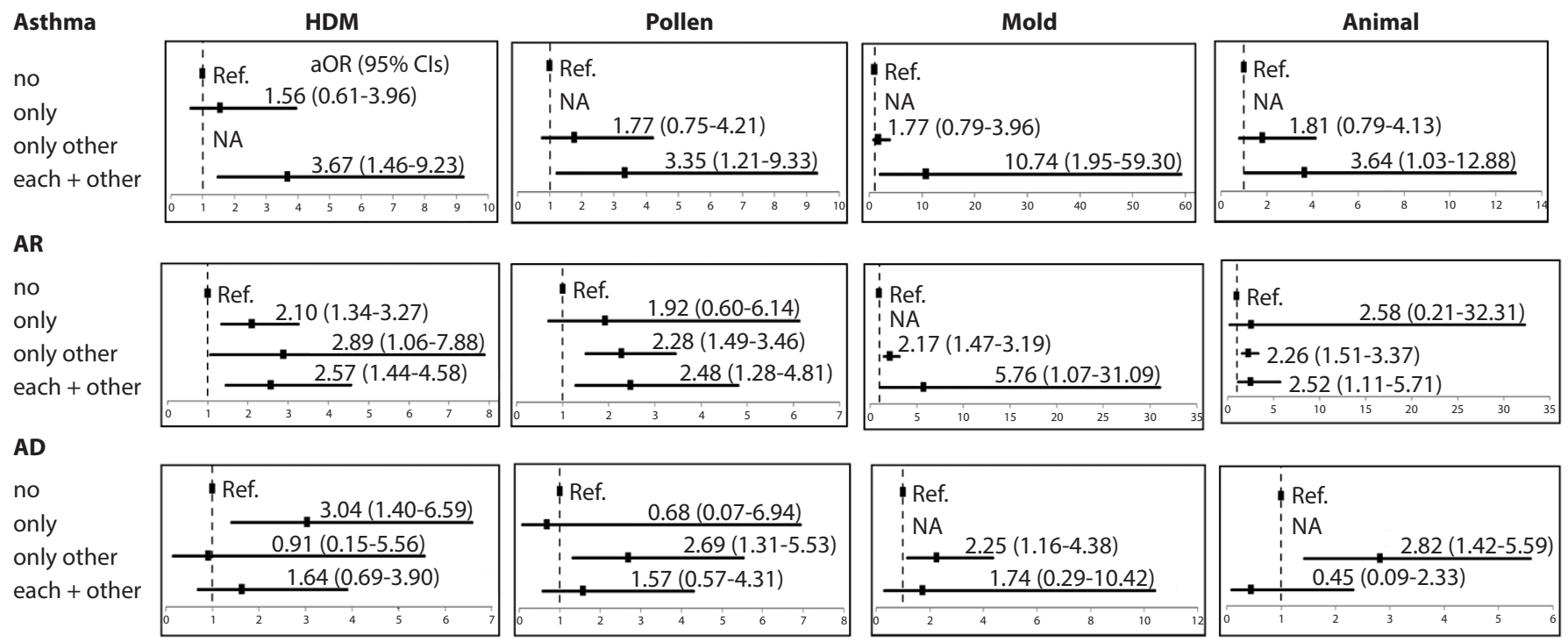

$\mathrm{BHR}<8 \mathrm{mg} / \mathrm{mL}$
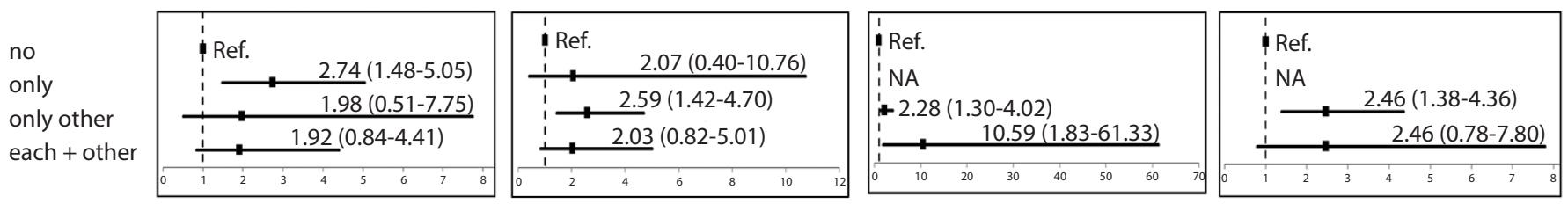

(B) Diagnosis by physicians in lifetime
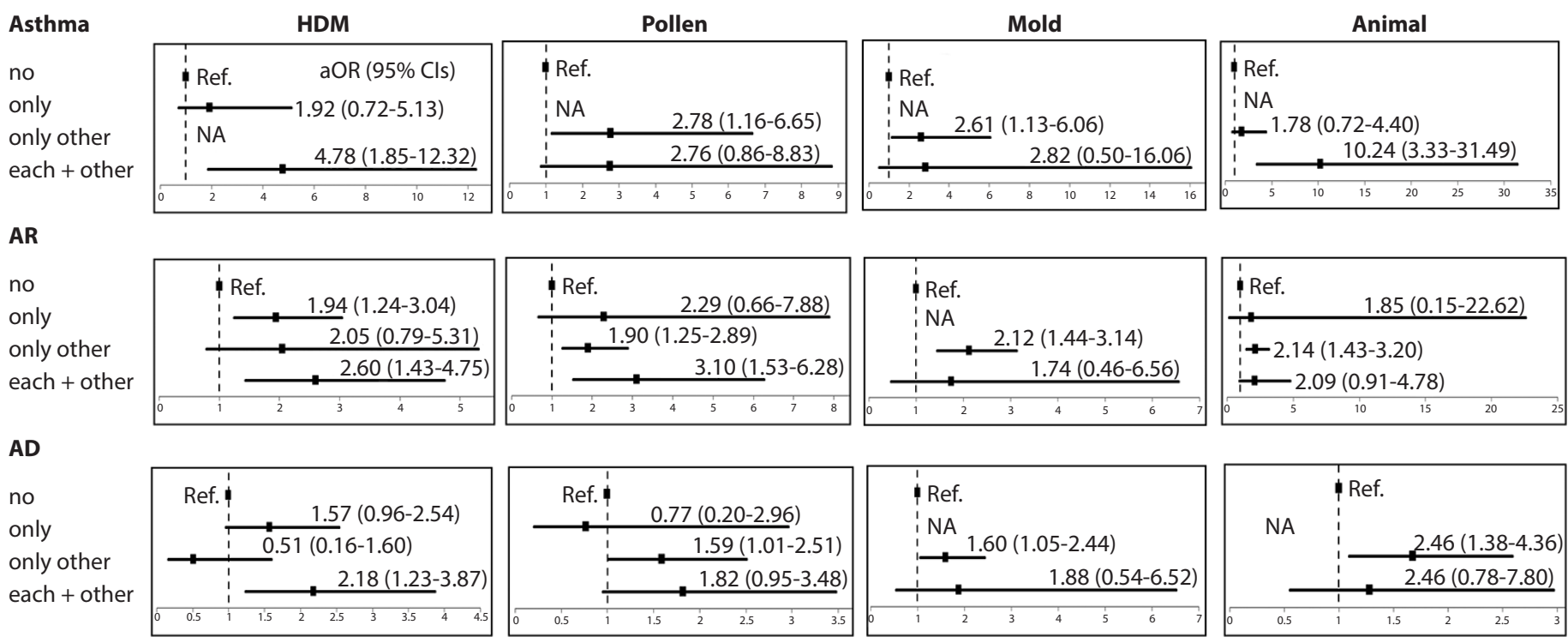

Figure 2. Association between sensitization patterns and symptoms of allergic diseases in the preceding 12 months (A) and lifetime diagnosis of allergic diseases by a physician (B).

'No', no sensitization to any allergen; 'only', sensitization to the index allergen only; 'only other', sensitization to allergens other than the index allergen; 'each + other', sensitization to both index allergens and more than one of the other allergens.

HDM included Der $\mathrm{p}$ and Der f; Pollen included alder, birch, oak, Japanese hop, mugwort, ragweed, and hazel; Mold included Alternaria and Aspergillus; Animal included dog dander and cat epithelium.

$\mathrm{AD}$, atopic dermatitis; aOR, adjusted odds ratio; AR, allergic rhinitis; CIs, confidence intervals; NA, not applicable; Ref., reference. 
Association between the number of sensitized allergen and allergic diseases

A higher number of sensitized allergens was found to be significantly associated with asthma symptoms in the preceding 12 months and lifetime diagnosis of asthma and AR (Figure 3). However, the number of sensitized allergens was not associated with the presence of $\mathrm{AD}$ symptoms in the preceding 12 months, lifetime diagnosis of $\mathrm{AD}$, or BHR.

\section{Association between the degree of sensitization and allergic diseases}

When we classified the degree of sensitization to indoor and outdoor allergens based on the highest sensitization grade for each allergen group, a higher degree of sensitization to indoor allergens was associated with symptoms in the preceding
12 months of asthma (aOR 5.67, 95\% CIs 1.69-19.00), AR (aOR 4.30, 95\% CIs 2.34-7.92), AD (aOR 3.39, 95\% CIs 1.288.94) and BHR (aOR 3.44, 95\% CIs 1.53-7.74). Sensitization to outdoor allergens was associated with symptoms in the recent 12 months of asthma (aOR 3.65, 95\% CIs 1.05-12.64) and AR (aOR 3.85, 95\% CIs 1.33-11.13), but not AD or BHR (Figure 4). In terms of lifetime diagnosis of any allergic disease, significantly increased associations were found between a higher degree of sensitization to indoor allergens and asthma (aOR 4.12, 95\% CIs 1.36-12.47 for sensitization higher than grade 4), $\mathrm{AR}$ (aOR 2.72, 95\% CIs 1.51-4.89 for sensitization higher than grade 4), and $\mathrm{AD}$ (aOR 2.06, 95\% CIs 1.13-3.76 for sensitization higher than grade 4). However, these associations were not observed between sensitization to outdoor allergens and lifetime diagnosis of any allergic disease.
(A) Asthma

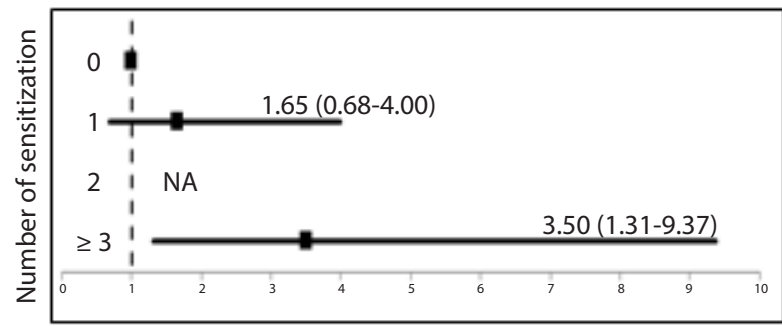

(B) AR

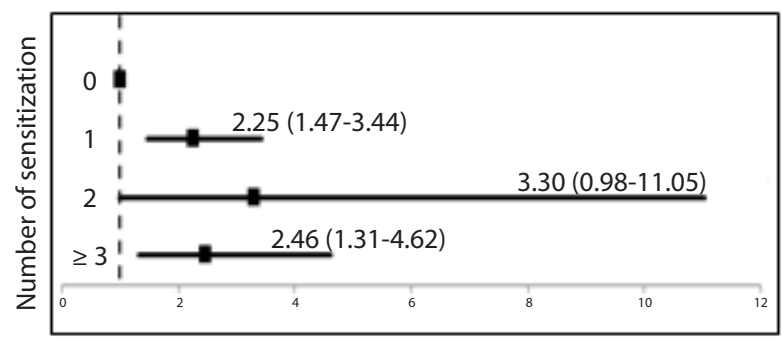

(C) AD

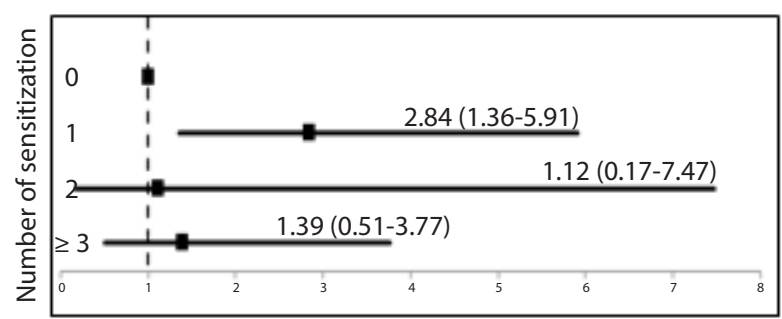

(D) $B H R<8 \mathrm{mg} / \mathrm{mL}$

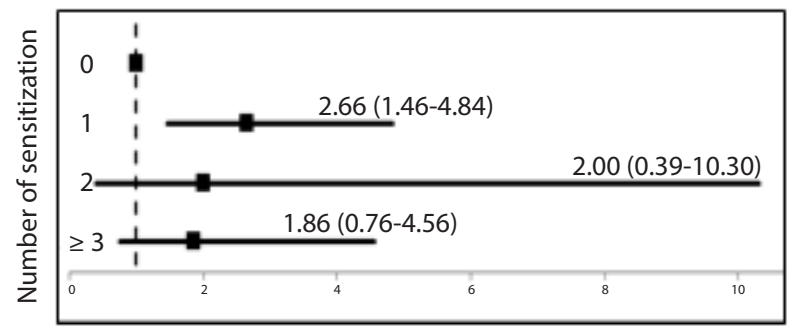

Diagnosis in lifetime
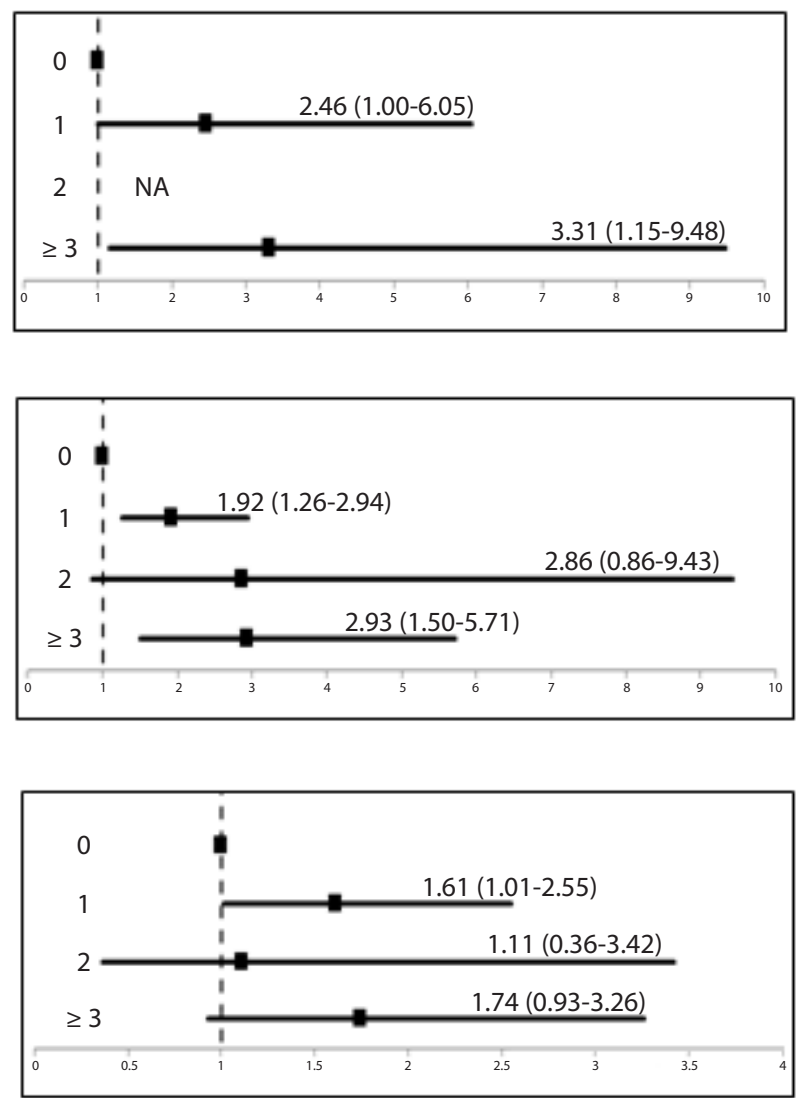

Figure 3. Adjusted odds ratio and $\mathbf{9 5 \%}$ confidence intervals for symptoms and lifetime diagnosis by a physician of allergic diseases according to the number of sensitized allergens at age 7 years. (A) Asthma. (B) AR. (C) AD. (D) BHR of less than 8 $\mathbf{m g} / \mathbf{m l}$.

$\mathrm{AD}$, atopic dermatitis; AR, allergic rhinitis; BHR, bronchial hyperresponsiveness; NA, not applicable. 
(A) Symptoms in the preceding 12 months

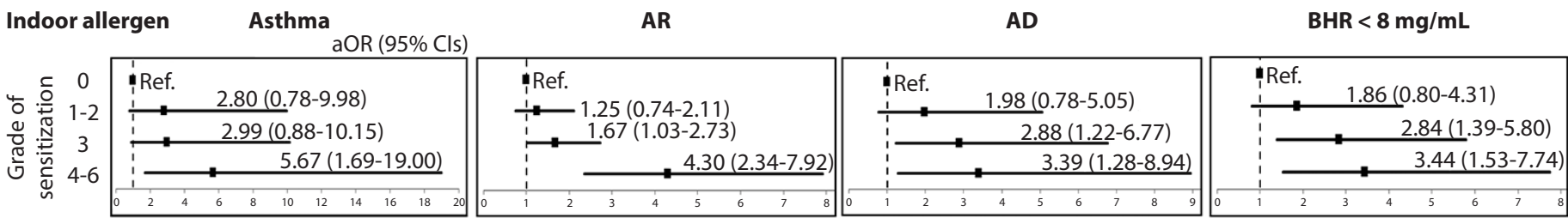

Outdoor allergen
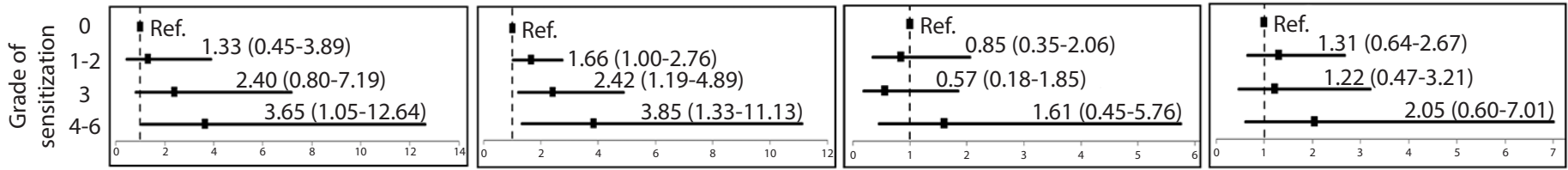

(B) Diagnosis by physicians in lifetime

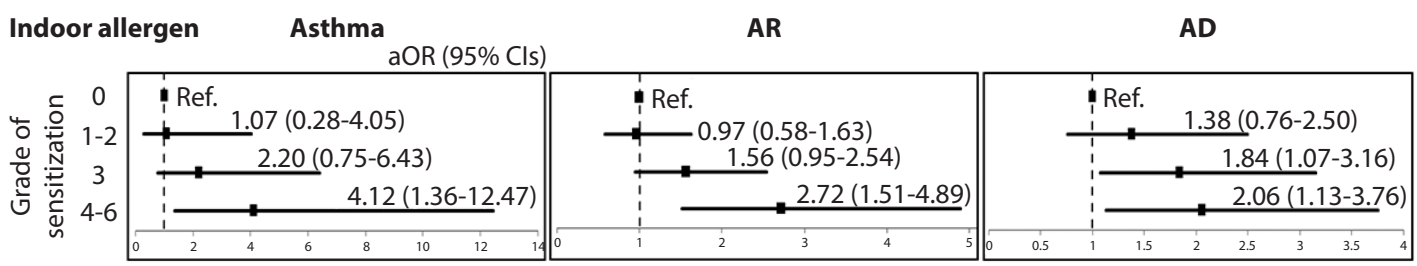

Outdoor allergen

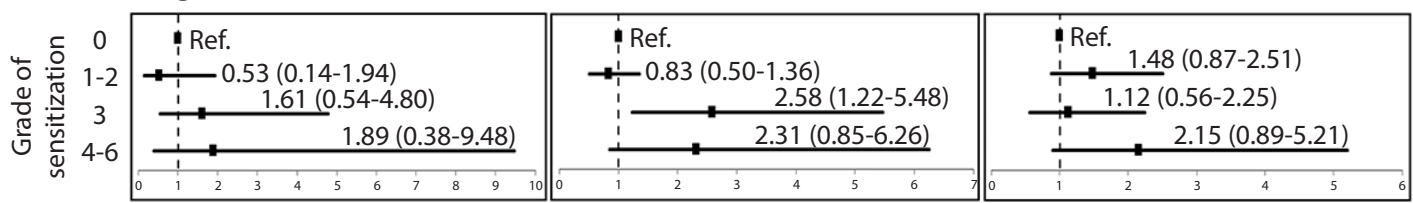

Figure 4. Adjusted odds ratio and $95 \%$ confidence intervals for symptoms in the preceding 12 months and lifetime diagnosis by a physician of allergic diseases according to severity of sensitization. (A) Association between symptoms of allergic diseases in the preceding 12 months and severity of sensitization. (B) Association between a diagnosis of specific allergic diseases in lifetime and the severity of sensitization.

$\mathrm{AD}$, atopic dermatitis; aOR, adjusted odds ratio; $\mathrm{AR}$, allergic rhinitis; $\mathrm{BHR}$, bronchial hyperresponsiveness; CI, confidence interval.

Indoor allergens included Der $p$, Der $f$, Aspergillus, dog dander, cat epithelium, and cockroach.

Outdoor allergens included Alternaria, alder, birch, oak, Japanese hop, mugwort, ragweed, and hazel.

Definition of sensitization grade: Grade 0 , ratio of allergen wheal to histamine $(\mathrm{R})=0$; grade $1,<1 / 2$; grade $2, \geq 1 / 2$ to $<1$; grade $3,1 \leq \mathrm{R}<2$; grade $4,2 \leq \mathrm{R}<3$; grade $5,3 \leq \mathrm{R}<4$; and grade $6, \mathrm{R} \geq 4$.

The selected allergens used to compare the grade of sensitization were based on the highest degree of sensitization in each allergen group.

\section{Discussion}

We have identified a sensitization rate of $46.4 \%$ in Korean children who were 7 years of age in 2015 with the most commonly sensitized allergens being $\operatorname{Der} p$ (38.0\%) and $\operatorname{Der} f$ (34.9\%). Sensitization to indoor allergens was associated with symptoms in the preceding 12 months and lifetime diagnosis of $\mathrm{AD}, \mathrm{AR}$ and asthma and BHR at 7 years of age, whereas sensitization to outdoor allergens was associated with symptoms in the preceding 12 months and lifetime diagnosis of AR. The number of sensitizations to common allergens was associated with symptoms in the preceding 12 months and lifetime diagnosis of asthma and AR, but not of $\mathrm{AD}$ and BHR. A higher degree of sensitization to indoor allergens was found to be significantly associated with symptoms in the preceding 12 months and lifetime diagnosis of asthma, AR, AD and BHR. However, higher degree sensitization to outdoor allergens was significantly associated with only symptoms of asthma and AR in the preceding 12 months. Based on these findings, we suggest that sensitization pattern including degree and number and types of allergens modifies the association between sensitization and allergic diseases. Our present study provides the reference data for the sensitization rate with commonly sensitized allergens and sensitization patterns in association with diverse allergic diseases in Korean children aged 7 years old from a general population-based birth cohort study. 
The sensitization rate of $46.4 \%$ in 7 years-old Korean children in our current cohort is higher than the previously reported rate of $40.9 \%$ in the same-aged subjects in $2010 . .^{12}$ This suggests that the sensitization rate in children is increasing over time. In our present study, HDM sensitizations were the most common, including $\operatorname{Der} f$ and $\operatorname{Der} p$. The similar findings have been reported by the previous studies performed in other countries and in Korea. ${ }^{9,12}$ US-based National Health and Nutrition Examination Surveys have indicated that the prevalence of sensitization to HDM has been increasing over time in children. ${ }^{9}$ in 2010 , the most common sensitizations were to $\operatorname{Der} f$ and $\operatorname{Der} p$ with rates of $32.4 \%$ and $32.1 \%$, respectively, in children aged 7 years. ${ }^{12}$ Based on the results of the previous and our present investigation (Der $p, 38.0 \%$ and $\operatorname{Der} f, 34.9 \%)$, sensitization to $\operatorname{Der} p$ and $\operatorname{Der} f$ seems to be showing an increasing incidence over time.

In our present study, sensitization to indoor allergens was found to be associated with diverse allergic diseases including asthma, $\mathrm{AR}$, and $\mathrm{AD}$ as well as $\mathrm{BHR}$, whereas sensitization to outdoor allergens was significantly associated only with AR. This positive association between sensitization to indoor allergens, such as HDM, and asthma in children has been reported previously. ${ }^{2,20}$ However, these previous studies did not consider simultaneous sensitization to different allergens. In our current study, we identified an association between independently sensitized allergen groups and allergic diseases. Among the diverse indoor allergens, sensitization to HDM was found to be independently associated with diverse allergic diseases including AR. A marked effect of mold sensitization on allergic diseases was also found when combined with sensitization to other allergens.

The complex interaction between sensitization patterns and the relevance of allergic diseases has been recently evaluated through phenotype studies. ${ }^{21-23}$ However, prior studies on atopy phenotypes are limited in identifying the association between independent sensitization patterns and allergic diseases due to the commonly sensitized allergens. ${ }^{23}$ To better identify an association between an independent sensitization to specific allergens and allergic diseases, we classified the common allergens into HDM, pollen, mold, and animal groups. Sensitization to HDM was independently associated with symptoms in the preceding 12 months of AR, AD, and BHR. Sensitization to HDM in combination with other groups of allergens increased the risk of recent symptoms and a lifetime diagnosis of asthma, but not sole sensitization to HDM. The independent sensitizations to pollen, mold, and animals were not associated with recent symptoms and lifetime diagnoses of any allergic disease, whereas combined sensitizations to other allergen groups was significantly associated with recent symptoms of asthma and AR. These findings may be explained by the relatively lower prevalence and weaker levels of independent sensitizations to pollen, mold and animal dander in this age group. Given that the recent studies on the association between allergic diseases and independently sensitized allergens are relatively lacking, the results of our study may provide important information on the association between sensitization patterns and allergic diseases that assists with their interpretation and prediction in children sensitized to specific inhalant allergens.
The previous studies on the association between BHR and sensitization in adults have indicated that sensitization to indoor allergens as well as multiple sensitizations are associated with $\mathrm{BHR},{ }^{7,24}$ but studies on these associations in children are lacking. In our present report, sensitizations to indoor allergens, especially higher degree sensitizations to indoor allergens, were associated with BHR in children and were more pronounced when the subjects were sensitized to common allergens in combination with mold. The results of our present study thus suggest that sensitization patterns might play a different role in development of allergic diseases as well as BHR.

Asignificant association was not found in our current study between number of sensitized allergens and BHR. We instead observed that degree of sensitizations to indoor allergens was associated with $\operatorname{BHR}(P=0.010$, Trend $P=0.002$, Pearson $\mathrm{R}=0.145$, Pearson $P=0.001$ ), whereas number as well as degree of sensitizations was associated with asthma in the present study. These findings might suggest that sensitization to indoor allergens in parallel with degree of sensitization, even if it is mono-sensitization, is associated with chronic airway inflammation, which is one of the requisite events in the onset of $\mathrm{BHR},{ }^{25}$ rather than sensitization in itself or number of sensitizations. The differences in sensitization patterns between asthma and BHR might be partially explained by the pathophysiologic differences between them or by other prerequisites for disease onset such as cytokine cascades. ${ }^{25}$ Although BHR is one of the key features of asthma, which is characterized by chronic airway inflammation combined with airway remodeling, ${ }^{26}$ it is also affected by the caliber of airway, as reflected by the smooth muscle contraction and edema. ${ }^{26-28}$ Multiple factors including a structurally disturbed bronchial epithelium and innate immunity are associated with asthma, regardless of $\mathrm{BHR},{ }^{29,30}$ whereas $\mathrm{BHR}$ is mainly associated with airway wall thickening. ${ }^{31}$ The differences between the sensitization pattern characteristics of asthma and BHR might be clinically relevant and future studies are thus needed to confirm the results of our present study and further elucidate the different pathophysiologies.

Although the role of sensitizations to inhalant allergens in the induction and persistence of $\mathrm{AD}$ has previously been unclear, ${ }^{32}$ a previous study has reported that the active avoidance of HDM exposure using bedcovers and a high filtration vacuum cleaner greatly decreases the severity of $\mathrm{AD}$ symptoms. ${ }^{33}$ An individual skin barrier dysfunction in susceptible subjects might affect the association between sensitization to $\mathrm{HDM}$ and $\mathrm{AD} .{ }^{34}$ Our current data suggest that sensitization to indoor allergens, but not outdoor allergens and mold, is associated with AD. In addition, we have observed that sensitization to HDM and its grade, but not number of sensitization, is more closely associated with $\mathrm{AD}$ in children. These findings may indicate that sensitizations to certain allergen types such as indoor allergens may in themselves play a crucial role in $\mathrm{AD}$, regardless of sensitization to multiple sensitization, distinct from other allergic diseases.

Genetic factors reflected in the presence of parental history of allergic disease, and high eosinophil and total serum IgE levels, also increase the risk of sensitization. In our present analyses, higher maternal education levels, which are often suggestive of a higher socioeconomic status, 
showed an increased risk of sensitization. This might be partially explained by changes in the composition of the human microbiota arising from immune system alterations caused by socioeconomic impacts on lifestyle, diet, and environment.

There are some limitations of our study of note. First, although this study was performed in a nationwide general population, the sample size was relatively small as a representation of the whole population in the 7-year-old age group. However, there were no significant differences in the demographic characteristics between the participants who visited each clinic center and those who did not. In addition, although we conducted a prospective birth cohort study with annual follow-ups, environmental factors and presence of allergic diseases were investigated by questionnaire, which could lead to a misdiagnosis of allergic diseases due to a subjective evaluation of the symptoms. However, ISAAC questionnaire has been used around the world to investigate the prevalence of allergic diseases. ${ }^{35}$ In our present study, we identified the association between BHR and sensitization patterns in children, as early as enforceable age with good performance for MCTs. In addition, the sensitization patterns might show regional variation, but we investigated regional differences in the sensitization patterns by adjustment due to limited sample sizes.

Despite these aforementioned shortcomings, our current analysis also has strengths such as use of a nationwide general population-based birth cohort study with a consideration of regional weights taken into account in our study design. Hence, our present findings can be generalized. In addition, although most prior general population-based studies on sensitization rates in children have included sensitization to aeroallergens, ${ }^{12}$ we included food allergens, although the sensitization rate was very low.

In summary, we report the sensitization rate of $46.4 \%$ in Korean children aged 7 years from a general-population based study. Sensitization patterns including allergen types, sensitization degree, and the number of different allergen sensitizations can differentially impact on diverse allergic diseases including AD, AR, asthma, and BHR. Our current findings will be helpful in interpreting sensitization patterns in association with allergic diseases. They may also prove useful for identifying the pathophysiologies and diverse phenotypes underlying different allergic diseases. Furthermore, our present data provide insights into future preventive strategies against specific sensitization patterns that are associated with specific allergic diseases.

\section{Potential conflicts of interest}

The authors declare no conflicts of interest in relation to this study.

\section{Financial disclosure}

This study was supported by a fund (2015-ER6601-00) from the Research of Korea Centers for Disease Control and Prevention, Panel Study of Korean Children (PSKC) of the Korea Institute of Child Care and Education (KICCE), and Basic Science Research Program through the National Research Foundation of Korea (NRF) funded by the Ministry of Education (2017M3A9F3043834).

\section{Author's contribution}

Lee E, Suh DI, Lee SY, Jung S, Yoon JS, Cho HJ, Kim YH, Yang SI, Kwon JW, Jang GC, Sun YH, Woo SI, Youn YS, Park KS, Cho HJ, Kook MH, Yi HR, Chung HL, Kim JH, Kim HY, Jung JA, Woo HO, Lee JK, Chang WS and Hong SJ designed the study, performed the analyses, interpreted the data, drafted this article and co-wrote and reviewed the manuscript.

\section{References}

1. Illi S, von Mutius E, Lau S, Niggemann B, Gruber C, Wahn U. Perennial allergen sensitisation early in life and chronic asthma in children: a birth cohort study. Lancet. 2006;368:763-70.

2. Arshad SH, Tariq SM, Matthews S, Hakim E. Sensitization to common allergens and its association with allergic disorders at age 4 years: A whole population birth cohort study. Pediatrics. 2001;108:art. no.-e33.

3. Alevizos M, Karagkouni A, Kontou-Fili K, Theoharides TC. A probable case report of stress-induced anaphylaxis. Ann Allergy Asthma Immunol. 2014;112:383-4.

4. Visitsunthorn N, Chaimongkol W, Visitsunthorn K, Pacharn P, Jirapongsananuruk $O$. Great flood and aeroallergen sensitization in children with asthma and/or allergic rhinitis. Asian Pac J Allergy Immunol. 2018;36:69-76.

5. Warm K, Hedman L, Lindberg A, Lotvall J, Lundback B, Ronmark E. Allergic sensitization is age-dependently associated with rhinitis, but less so with asthma. J Allergy Clin Immunol. 2015;136:1559-65 e2.

6. Leung R, Ho P, Lam CW, Lai CK. Sensitization to inhaled allergens as a risk factor for asthma and allergic diseases in Chinese population. J Allergy Clin Immunol. 1997;99:594-9.

7. Kerkhof M, Postma DS, Schouten JP, de Monchy JG. Allergic sensitization to indoor and outdoor allergens and relevance to bronchial hyperresponsiveness in younger and older subjects. Allergy. 2003;58: 1261-7.

8. Vartiainen E, Petays T, Haahtela T, Jousilahti P, Pekkanen J. Allergic diseases, skin prick test responses, and IgE levels in North Karelia, Finland, and the Republic of Karelia, Russia. J Allergy Clin Immunol. 2002;109: 643-8.

9. Salo PM, Arbes SJ, Jr., Jaramillo R, Calatroni A, Weir CH, Sever ML, et al. Prevalence of allergic sensitization in the United States: results from the National Health and Nutrition Examination Survey (NHANES) 2005-2006. J Allergy Clin Immunol. 2014;134:350-9.

10. Nissen SP, Kjaer HF, Host A, Nielsen J, Halken S. The natural course of sensitization and allergic diseases from childhood to adulthood. Pediatr Allergy Immunol. 2013;24:549-55.

11. Lee E, Lee SH, Kim YH, Cho HJ, Yoon J, Yang SI, et al. Association of atopy phenotypes with new development of asthma and bronchial hyperresponsiveness in school-aged children. Ann Allergy Asthma Immunol. 2017;118:542-50 e1.

12. Kim J, Hahm MI, Lee SY, Kim WK, Chae Y, Park YM, et al. Sensitization to aeroallergens in Korean children: a population-based study in 2010. J Korean Med Sci. 2011;26:1165-72.

13. Yoo B, Park Y, Park K, Kim H. A 9-year trend in the prevalence of allergic disease based on national health insurance data. J Prev Med Public Health. 2015;48:301-9.

14. Kang SY, Song WJ, Cho SH, Chang YS. Time trends of the prevalence of allergic diseases in Korea: A systematic literature review. Asia Pac Allergy. 2018;8:e8.

15. Boulet LP, Turcotte H, Laprise C, Lavertu C, Bedard PM, Lavoie A, et al. Comparative degree and type of sensitization to common indoor and outdoor allergens in subjects with allergic rhinitis and/or asthma. Clin Exp Allergy. 1997;27:52-9.

16. Jacob B, Ritz B, Gehring U, Koch A, Bischof W, Wichmann HE, et al. Indoor exposure to molds and allergic sensitization. Environ Health Perspect. 2002;110:647-53.

17. van der Valk JP, Gerth van Wijk R, Hoorn E, Groenendijk L, Groenendijk IM, de Jong NW. Measurement and interpretation of skin prick test results. Clin Transl Allergy. 2015;6:8.

18. Crapo RO, Casaburi R, Coates AL, Enright PL, Hankinson JL, Irvin CG, et al. Guidelines for methacholine and exercise challenge testing-1999. This official statement of the American Thoracic Society was adopted by the ATS Board of Directors, July 1999. Am J Respir Crit Care Med. 2000;161:309-29. 
19. Hong SJ, Kim SW, Oh JW, Rah YH, Ahn YM, Kim KE, et al. The validity of the ISAAC written questionnaire and the ISAAC video questionnaire (AVQ 3.0) for predicting asthma associated with bronchial hyperreactivity in a group of 13-14 year old Korean schoolchildren. J Korean Med Sci. 2003;18:48-52.

20. Ruggieri S, Drago G, Longo V, Colombo P, Balzan M, Bilocca D, et al. Sensitization to dust mite defines different phenotypes of asthma: A multicenter study. Pediatr Allergy Immunol. 2017;28:675-82.

21. Simpson A, Tan VY, Winn J, Svensen M, Bishop CM, Heckerman DE, et al. Beyond atopy: multiple patterns of sensitization in relation to asthma in a birth cohort study. Am J Respir Crit Care Med. 2010;181:1200-6.

22. Schoos AM, Chawes BL, Melen E, Bergstrom A, Kull I, Wickman M, et al. Sensitization trajectories in childhood revealed by using a cluster analysis. J Allergy Clin Immunol. 2017;140:1693-9.

23. Schoos AM, Chawes BL, Rasmussen MA, Bloch J, Bonnelykke K, Bisgaard H. Atopic endotype in childhood. J Allergy Clin Immunol. 2016;137:844-51 e4.

24. Plaschke P, Janson C, Norrman E, Bjornsson E, Ellbjar S, Jarvholm B. Association between atopic sensitization and asthma and bronchial hyperresponsiveness in swedish adults: pets, and not mites, are the most important allergens. J Allergy Clin Immunol. 1999;104:58-65.

25. Samitas K, Carter A, Kariyawasam HH, Xanthou G. Upper and lower airway remodelling mechanisms in asthma, allergic rhinitis and chronic rhinosinusitis: The one airway concept revisited. Allergy. 2018;73:993-1002.

26. Brannan JD, Lougheed MD. Airway hyperresponsiveness in asthma: mechanisms, clinical significance, and treatment. Front Physiol. 2012;3:460.
27. Kim YH, Lee E, Cho HJ, Yang SI, Jung YH, Kim HY, et al. Association between menarche and increased bronchial hyper-responsiveness during puberty in female children and adolescents. Pediatr Pulmonol. 2016;51: 1040-7.

28. Sluiter HJ, Koeter GH, de Monchy JG, Postma DS, de Vries K, Orie NG. The Dutch hypothesis (chronic non-specific lung disease) revisited. Eur Respir J. 1991;4:479-89.

29. Loxham M, Davies DE. Phenotypic and genetic aspects of epithelial barrier function in asthmatic patients. J Allergy Clin Immunol. 2017;139:1736-51.

30. Holgate ST, Lackie P, Wilson S, Roche W, Davies D. Bronchial epithelium as a key regulator of airway allergen sensitization and remodeling in asthma. Am J Respir Crit Care Med. 2000;162:S113-7.

31. Niimi A, Matsumoto H, Takemura M, Ueda T, Chin K, Mishima M. Relationship of airway wall thickness to airway sensitivity and airway reactivity in asthma. Am J Respir Crit Care Med. 2003;168:983-8.

32. Friedmann PS. The role of dust mite antigen sensitization and atopic dermatitis. Clin Exp Allergy. 1999;29:869-72.

33. Tan BB, Weald D, Strickland I, Friedmann PS. Double-blind controlled trial of effect of housedust-mite allergen avoidance on atopic dermatitis. Lancet. 1996;347:15-8.

34. Kubo A, Nagao K, Amagai M. Epidermal barrier dysfunction and cutaneous sensitization in atopic diseases. J Clin Invest. 2012;122:440-7.

35. Lewis S. ISAAC--a hypothesis generator for asthma? International Study of Asthma and Allergies in Childhood. Lancet. 1998;351:1220-1. 